\title{
Integrative systems of genomic risk markers for cancer and other diseases: future of predictive medicine
}

This article was published in the following Dove Press journal:

Cancer Management and Research

22 May 2012

Number of times this article has been viewed

\author{
Arseniy E Yuzhalin \\ Anton G Kutikhin \\ Department of Epidemiology, \\ Kemerovo State Medical Academy, \\ Kemerovo, Russian Federation
}

Correspondence: Arseniy E Yuzhalin Voroshilova Street 22a, Kemerovo, 650029, Russian Federation Tel +7923525 I 517

Fax +7 3842360546

Email yuzhalin@gmail.com
Abstract: This paper focuses on genomic predictive biomarkers in medicine. It is known that single nucleotide polymorphisms of genes involved in the pathogenesis of various diseases, including cancer, can serve as indicators of risk warranting further diagnostics, screening, and early prevention measures. However, although ten million single nucleotide polymorphisms have been identified to date, the majority of them do not appear to have a relationship with risk of development of pathogenic processes in the human body. The concept of selection of significant disease-associated biomarkers is proposed. Also, future development of integrative systems of genomic risk markers is discussed.

Keywords: biomarkers, cancer, single nucleotide polymorphisms, cancer prevention

\section{Genomic biomarkers in medicine}

One of the most hazardous public health problems is cancer. More than seven million deaths from cancer were registered worldwide in $2008 .{ }^{1}$ However, annual death rates have been decreasing slowly since 1990 in men and since 1991 in women. ${ }^{1}$ This progress is largely as a result of intensive development of modern preventative measures against cancer, the efficiency of which is growing every year. Obviously, prevention of a disease is much easier than its treatment, and so the problem of cancer prevention is one of the most basic issues when combating the burden of the disease.

Novel approaches in health care are moving towards the model of "personalized medicine". ${ }^{2}$ Advances in health care are growing annually, as well as their social relevance. Diagnostic tests and targeted therapy have become common. However, in spite of novel improvements in screening and prevention modalities, the prognosis of patients with many diseases, including cancer, remains poor. Thus, modern molecular biology and medicine are concerned with developing more and more novel genomic markers with predictive, therapeutic, and prognostic significance. ${ }^{3}$ Several markers may evaluate the predisposition of a given person to one or another disease with a certain degree of accuracy based on the results of a simple blood test. Widespread application of these tests can reveal risk groups in populations, and thereafter, a complex of preventive measures among risk-group subjects may be conducted. Moreover, the abovementioned genomic markers can be identified in the perinatal period, so the choice between "include" or "not to include" in the risk group on the basis of these systems can be made very early, and, consequently, preventive measures can have maximal efficacy. As a result, integrative systems of predictive genomic markers, once defined, 
will allow future generations to be informed and forewarned about their risks of and predispositions to certain diseases.

Therefore, discovery and development of predictive, therapeutic, or prognostic markers is the primary problem of biomedicine at the present time. However, the critical barrier to progress in this field is that it is not always easy to find an effective genomic marker that is specifically associated with a particular disease. ${ }^{3}$ One of the most widespread and important genomic markers is the single nucleotide polymorphism, which represents a variation in DNA sequence, when a single nucleotide differs between members of a biological species or paired chromosomes in an individual. The presence of such a substitution in DNA sequence may often cause a deviation of protein function and/or lead to disruption of exonic splicing enhancer sequences. ${ }^{4}$ Single nucleotide polymorphisms may lead to changes in transcription factors and vary the efficiency of gene expression, as well as introduce an alternative translation initiation codon that may lead to downregulation of the wild-type transcript. ${ }^{4}$ It is known that single nucleotide polymorphisms can cause instability of exonic mRNA as well. ${ }^{5}$ Because they often have relatively substantial functional consequences and a potentially high incidence in a population, single nucleotide polymorphisms can be very informative and are extensively used in studies of their association with risk for many diseases, including cancer. Hence, it is feasible to say that identification of single nucleotide polymorphisms as markers of cancer predisposition is a convenient, simple, and effective way to identify and treat various malignancies in the earliest stages.

Over ten million single nucleotide polymorphisms have been identified to date, and the majority of them may not have any association with risk of occurrence or features of development of pathogenic processes in the human body. ${ }^{6}$ Therefore, a major question arises as to which genes and which polymorphisms should be selected for further investigation of their possible associations with cancer. It is necessary to mention briefly the major families of proteins and enzymes for which genes and functional single nucleotide polymorphisms are currently being investigated for their association with cancer risk.

\section{Antioxidant defense system}

A great number of carcinogens promote the generation of so-called reactive oxygen species, which cause damage to DNA and may therefore lead to development of cancer. DNA damage caused by reactive oxygen species is termed "oxidative stress". The most common reactive oxygen species are superoxide radicals, hydroxide radicals, peroxyl radicals, nitric oxide, alkoxyl radicals, and hydrogen peroxide. Nevertheless, human cells are protected against oxidative stress by an interacting network of antioxidant enzymes, including catalases, superoxide dismutases, glutathione peroxidases, peroxiredoxins, paraoxonases, and many others. The role of these enzymes is to break down dangerous reactive compounds before they reach a strand of DNA. Hence, effective and proper functioning of antioxidant defense enzymes is the first barrier to carcinogens entering the living cell.

\section{DNA repair system}

In spite of antioxidant defense enzymes, the genome of each human cell acquires more than one million molecular lesions every day. ${ }^{7}$ Protection of cells against accumulation of numerous mutations is carried out by DNA repair enzymes. These enzymes are able to recognize single-strand and double-strand breaks and repair them. Moreover, DNA repair enzymes correct the erroneous insertion, deletion, and misincorporation of bases that occurs during the recombination and replication of DNA. Thereby, DNA repair enzymes maintain a more or less stable state of the genome, and the rate of successful DNA repairs reflects the best chances for the cell to avoid occurrence of an oncogenic mutation.

\section{Apoptotic pathways}

In the event that accumulation of DNA damage becomes large due to severe stress, or the DNA repair system is poor, the cell then undergoes so-called programmed cell death or apoptosis. Apoptosis occurs because some cells become cancerous owing to the large amount of accumulated errors. Because they pose a potential threat to the organism, it is better to kill them for the better good. Thereby, in the context of cancer, apoptosis greatly decreases the likelihood of a cell becoming cancerous. ${ }^{8}$ Initiation of apoptosis is controlled by numerous regulatory and adaptor proteins, which are activated by various intracellular and/or extracellular signals. It is known that inhibition of apoptosis plays an important role in the development of cancer. ${ }^{9}$ Moreover, mutations in cycle-regulating genes, such as ras, c-myc, or p53, may lead to development of cancer or a significantly increased cancer risk. $^{10-12}$

\section{Pattern recognition receptors}

It is well known that some viruses can induce cancer because they carry oncogenes in their genomes. ${ }^{10}$ In other cases, viruses may incorporate their genome near the proto-oncogene, putting it under the control of the viral 
transcriptional promoter. ${ }^{10}$ In addition, it is necessary to note that any infectious agent provoking inflammation can initiate cancer as well, because the presence of a prolonged inflammatory response is accompanied by severe cellular stress, and consequently may lead to accumulation of DNA damage, mutations, and eventually promote cancer. Pattern recognition receptors directly recognize common antigen determinants of virtually all classes of pathogens (so-called pathogen-associated molecular patterns) and initiate an immune response against them via specific intracellular signaling pathways. Pattern recognition receptors include families of Toll-like receptors, NOD-like receptors, C-type lectin receptors, and RIG-I-like receptors. Their work protects the organism from infectious agents and may prevent, inhibit, or block carcinogenesis, whilst disrupted functioning of these pattern recognition receptors may allow infectious agents or tumor cells to avoid recognition by the immune system and, consequently, not be eliminated. ${ }^{13}$ Notably, such pattern recognition receptor activation may sometimes promote carcinogenesis, creating a proinflammatory microenvironment (via the action of respective cytokines) that is favorable for tumor progression and development of chemoresistance. ${ }^{13}$ Thereby, pattern recognition receptors play a significant role in the development of cancer, and maintaining a balance between low and high pattern recognition receptor activity greatly decreases the risk of a cancer developing.

\section{Immune system}

Despite all the above-mentioned mechanisms of protection, cancers do occur. When there is a tumor in the organism, the only thing that may help is the immune response. There is a huge amount of evidence indicating that the immune system plays a key role in the battle of the organism against cancer., ${ }^{70}$ Tumor cells express their own antigens which cause immune cells to recognize cancer and eliminate it. However, tumor cells often have a reduced number of major histocompatibility complex class I molecules on their surface, thus avoiding detection by killer $\mathrm{T}$ cells. ${ }^{14}$ The crucial role in anticancer defense belongs to cytokines, a diverse multifunctional group of proteins that facilitate communication between immune cells, control genes, regulate transcription factors, and govern the inflammation, differentiation, proliferation, and secretion of antibodies. ${ }^{7}$ The most important cytokine classes are interleukins, interferons, and tumor necrosis factors. It is important to note that the harmonious, coordinated, and smooth functioning of cytokines in many respects determines the effectiveness of the anticancer immune response. In some cases, incorrect functioning of cytokines (frequently caused by underexpression or overexpression of certain cytokine genes) directly favors development of cancer. For instance, a shift in the balance between the activity of proinflammatory and anti-inflammatory interleukins may cause prolonged inflammation, and therefore promote neoplastic transformation..$^{15,16}$ Another example is that it is known that interleukin-17 possesses strong angiogenic activity due to its ability to inhibit angiostasis and enhance secretion of angiogenic chemokines; therefore, overexpression of interleukin-17 may contribute significantly to the massive blood supply to the tumor. ${ }^{17}$

In general, all the above-mentioned systems protect against the occurrence or progression of cancer in a large number of cases, and inherited variations in genes that encode the above-named proteins and enzymes are responsible for individual susceptibility to cancer. The next question that arises is how to select single nucleotide polymorphisms that can potentially be associated with cancer?

\section{Concept of selection}

Gene polymorphism may be included on the short list for further oncogenomic studies if:

- Single nucleotide polymorphisms lead to substantial functional consequences at the molecular level (eg, it strongly affects transcription, splicing, translation, stability, and transport of pre-mRNA, mRNA, noncoding RNA, or protein encoding by the gene, or noticeably influences signaling of synthesized protein)

- Single nucleotide polymorphism is frequent in populations

- Functional consequences of the single nucleotide polymorphism are significantly associated with a condition that substantially increases the risk of cancer due to deregulation of systems listed above

- Single nucleotide polymorphism is associated with risk of cancer in population studies.

Gene polymorphism can also be included on the extended list if it is characterized by more subtle functional alterations in the gene that still result in qualitative or quantitative alterations of the encoding protein (or noncoding RNA). One question that arises immediately is how to distinguish "substantial" and more "subtle" functional changes at the molecular level? It seems to be difficult to answer this question only on the basis of the general principles of molecular biology, because even the smallest alteration in the structure of one gene may have critical consequences, while for another gene the converse can be true. Therefore, an assessment of the "power" of functional alteration should be individualized 
for each gene, and even if conclusions obtained in various investigations do differ, these discrepancies would not distort the general picture, ie, if the polymorphism has "serious" functional consequences according to the results of an isolated study, it should definitely be added to the short list until these conclusions are disproven. In any case, the general value of creating such short and extended lists of prescriptive polymorphisms is that they can overcome the difficulties related to these complications. It is important to note that many polymorphisms can be simply in linkage disequilibrium with truly functional variants, and fundamental investigations are needed to determine if they are merely markers of association or indeed causal variants. All polymorphisms that are only in linkage disequilibrium with functional ones should be excluded from both lists.

The results of various population studies devoted to the investigation of association between gene polymorphisms and cancer may also differ, and possible reasons for these disparities include an inadequate sample size, and differences in age, gender, ethnic, racial, and clinicopathological characteristics, in prevalence of the infectious agent relevant to the disease under investigation in cases and control groups, in other bacterial, host, or environmental factors, in the immune response caused by a specific ligand, in stratification, and in methods of diagnostics of cancer or chronic inflammatory conditions, and genotyping methods, as well as chance. In addition, studies for which negative results are obtained tend not to be published (the so-called "file drawer" effect), which may create a significant bias and distort the picture observed at the present time.

To distinguish the impact of chronic inflammatory conditions from the contribution of other mechanisms to the association between gene polymorphisms and cancer risk, stratification of cases and controls by infectious agent status and chronic inflammation status should be mandatory in future studies devoted to this question. Sample size should be sufficient, and this depends on the frequency of the target polymorphism; if the frequency is high, the sample size can be less than in studies where the frequency is low. It is also important to note that there are two main components determining the importance of single nucleotide polymorphisms in integrative systems of genomic risk markers that can be used in programs for cancer prevention, ie, the value of the odds ratio between cases and controls (in the whole population as well as in subgroups) and the prevalence of the polymorphism in the population, and both these components may vary in different geographic regions. Moreover, it is desirable to develop not just one general program, but a number of individual programs for different countries, populations, and environmental conditions.

\section{Looking into the future}

It is certainly true that further investigations of single nucleotide polymorphisms will provide new and robust data for the molecular epidemiology of cancer. In the event that an association between certain single nucleotide polymorphisms of and increased or decreased cancer risk is revealed and confirmed by various case-control studies in different populations, then these polymorphisms should be established as valid predictive markers of cancer. In the future, such genomic cancer risk markers should be unified in the general integrative systems for each cancer type, population, and/or subgroups of the population, and should be used in various programs for cancer prevention. Generally, these programs would consist of the following steps:

- Maximal early (perinatal) determination of risk groups (high, moderate, and low), and further stratification of these risk groups into intermediate categories

- Development of preventive measures for these risk groups.

In addition, development of an integrative system of genomic risk markers would provide a theoretical basis for the creation of a specific microchip including all risk factors, which will greatly simplify determination of cancer risk. Furthermore, the above-mentioned concept should be true not only for cancer, but also for other human diseases. It may also be useful to create an international electronic open-access database of short and extended lists of polymorphisms of each gene for every disease to stratify prospective polymorphisms for genomic investigation. Finally, this strategy may lead to breakthroughs for preventive interventions in the field of the molecular epidemiology of cancer and other diseases.

\section{Acknowledgments}

The authors would like to express their gratitude to the anonymous referees who helped improve this paper.

\section{Disclosure}

The authors report no conflicts of interest in this work.

\section{References}

1. Jemal A, Bray F, Center MM, Ferlay J, Ward E, Forman D. Global cancer statistics. CA Cancer J Clin. 2011;61:69-90.

2. Zineh I, Huang SM. Biomarkers in drug development and regulation: a paradigm for clinical implementation of personalized medicine. Biomark Med. 2011;5:705-713.

3. On beyond GWAS. Editorial. Nature Genetics. 2010;42(7):551. 
4. Sachidanandam R, Weissman D, Schmidt SC, Kakol JM, Stein LD, Marth G. A map of human genome sequence variation containing 1.42 million single nucleotide polymorphisms. Nature. 2001;409:928-933.

5. Duan J, Wainwright MS, Comeron JM, Saitou N, Sanders AR, Gelernter J. Synonymous mutations in the human dopamine receptor D2 (DRD2) affect mRNA stability and synthesis of the receptor. Hum Mol Genet. 2003;12:205-216.

6. Tsigris C, Chatzitheofylaktou A, Xiromeritis C, Nikiteas N, Yannopoulos A. Genetic association studies in digestive system malignancies. Anticancer Res. 2007;27:3577-3587.

7. Lodish H, Berk A, Matsudaira P, et al. Molecular Biology of the Cell, 5th ed. New York, NY: WH Freeman; 2004.

8. Thompson CB. Apoptosis in the pathogenesis and treatment of disease. Science. 1995;267:1456-1462.

9. Varfolomeev E, Vucic D. Inhibitor of apoptosis proteins: fascinating biology leads to attractive tumor therapeutic targets. Future Oncol. 2011;7:633-648.

10. Weinberg RA. The Biology of Cancer. New York, NY: Garland Science; 2007.
11. Piao JM, Kim HN, Song HR, et al. p53 codon 72 polymorphism and the risk of lung cancer in a Korean population. Lung Cancer. 2011;S73: 264-267.

12. Huang CY, Su CT, Chu JS, et al. The polymorphisms of P53 codon 72 and MDM2 SNP309 and renal cell carcinoma risk in a low arsenic exposure area. Toxicol Appl Pharmacol. 2011;257:349-355.

13. Kutikhin AG, Yuzhalin AE. Inherited variation in pattern recognition receptors and cancer: dangerous liaisons? Cancer Manag Res. 2012;4:31-38.

14. Seliger B, Ritz U, Ferrone S. Molecular mechanisms of HLA class I antigen abnormalities following viral infection and transformation. Int J Cancer. 2006;118:129-138.

15. McLean MH, El-Omar EM. Genetics of inflammation in the gastrointestinal tract and how it can cause cancer. Recent Results Cancer Res. 2011;185:173-183.

16. Neurath MF, Finotto S. IL-6 signaling in autoimmunity, chronic inflammation and inflammation-associated cancer. Cytokine Growth Factor Rev. 2011;22:83-89.

17. Murugaiyan G, Saha B. Protumor vs antitumor functions of IL-17. J Immunol. 2009;183:4169-4175.
Cancer Management and Research

\section{Publish your work in this journal}

Cancer Management and Research is an international, peer-reviewed open access journal focusing on cancer research and the optimal use of preventative and integrated treatment interventions to achieve improved outcomes, enhanced survival and quality of life for the cancer patient The journal welcomes original research, clinical \& epidemiological

\section{Dovepress}

studies, reviews \& evaluations, guidelines, expert opinion \& commentary, case reports \& extended reports. The manuscript management system is completely online and includes a very quick and fair peerreview system, which is all easy to use. Visit http://www.dovepress.com/ testimonials.php to read real quotes from published authors. 